\title{
Bounds for Stop-Loss Premiums of Stochastic Sums (with Applications to Life Contingencies)
}

\author{
Tom Hoedemakers* ${ }^{* \ddagger}$ Grzegorz Darkiewicz ${ }^{\dagger}$ Griselda Deelstra ${ }^{\S}$ \\ Jan Dhaene ${ }^{\dagger}$ Michèle Vanmaeleף
}

\begin{abstract}
In this paper we present in a general setting lower and upper bounds for the stop-loss premium of a (stochastic) sum of dependent random variables. Therefore, use is made of the methodology of comonotonic variables and the convex ordering of risks, introduced by Kaas et al. (2000) and Dhaene et al. (2002a, 2002b), combined with actuarial conditioning. The lower bound approximates very accurate the real value of the stop-loss premium. However, the comonotonic upper bounds perform rather badly for some retentions. Therefore, we construct sharper upper bounds based upon the traditional comonotonic bounds. Making use of the ideas of Rogers and Shi (1995), the first upper bound is obtained as the comonotonic lower bound plus an error term. Next this bound is refined by making the error term dependent on the retention in the stop-loss premium. Further, we study the case that the stop-loss premium can be decomposed into two parts. One part which can be evaluated exactly and another part to which comonotonic bounds are applied. As an application we study the bounds for the stop-loss premium of a random variable representing the stochastically discounted value of a series of cash flows with a fixed and stochastic time horizon. The paper ends with numerical examples illustrating the presented approximations. We apply the proposed bounds for life annuities, using Makeham's law, when also the stochastic nature of interest rates is taken into account by means of a Brownian motion.
\end{abstract}

Keywords: stop-loss premium, life annuity, comonotonicity, stochastic time horizon.

\footnotetext{
*Corresponding author. E-mail address: Tom.Hoedemakers@econ.kuleuven.ac.be

${ }^{\dagger}$ Department of Applied Economics, Catholic University Leuven, Naamsestraat 69, 3000 Leuven, Belgium

${ }^{\ddagger}$ University Center of Statistics, W. de Croylaan 54, 3001 Heverlee, Belgium

${ }^{\S}$ Department of Mathematics, ISRO and ECARES, Université Libre de Bruxelles, Boulevard du Triomphe, 2, CP 210, 1050 Brussels, Belgium

`Department of Applied Mathematics and Computer Science, Ghent University, Krijgslaan 281, S9, 9000 Gent, Belgium
} 


\section{Introduction}

An insurance risk is typically described by a random variable $X \geq 0$. Here $X$ can represent for example a single insurance claim, an aggregate (discounted) value of future claims for an individual contract or an aggregate value for a portfolio of insurance contracts over a given period. One of the most important tasks of actuaries is to assess the degree of dangerousness of a risk $X$ - either by finding the (approximate) distribution or at least by summarizing its properties quantitatively by means of risk measures to determine an insurance premium or a sufficient reserve with solvency margin.

A stop-loss premium $\pi(X, d)=\mathrm{E}\left[(X-d)_{+}\right]=\mathrm{E}[\max (0, X-d)]$ is one of the most important risk measures. The retention $d$ is usually interpreted as an amount retained by an insured (or an insurer) while an amount $X-d$ is ceded to an insurer (or a reinsurer). In this case $\pi(X, d)$ has a clear interpretation of a pure insurance (reinsurance) premium.

Another practical application of stop-loss premiums is the following: Suppose that a financial institution faces a risk $X$ to which a capital $K$ is allocated. Then the residual risk $R=(X-K)_{+}$ is a quantity of concern to the society and regulatories. Indeed, it represents the pessimistic case when the random loss $X$ exceeds the available capital. The value $\mathrm{E}[R]$ is often referred to as the "expected shortfall".

It is not always straightforward to compute stop-loss premiums. In the actuarial literature a lot of attention has been devoted to determine bounds for stop-loss premiums in case only partial information about the claim size distribution is available (e.g. De Vylder and Goovaerts (1982), Jansen et al. (1986), Hürlimann (1996, 1998) among others).

Other types of problems appear in the case of sums of random variables $\mathbb{S}=X_{1}+\cdots+X_{n}$ when full information about marginal distributions is recognized but the dependency structure is not known. In Dhaene et al. (2002a, 2002b) it was shown that the upper bound $\mathbb{S}^{c}$ of the sum $\mathbb{S}$ in so called stop-loss order sense can be derived by replacing the unknown copula of the random vector $\left(X_{1}, X_{2}, \ldots, X_{n}\right)$ by the most dangerous comonotonic copula. They propose also the lower bound $\mathbb{S}^{\ell}$ obtained through conditioning. Such an approach allows to determine analytical bounds for stop-loss premiums.

In practical applications the comonotonic upper bound seems to be useful only in the case of a very strong dependency between summands. Even then the bounds for stop-loss premiums provided by the comonotonic approximation are often not satisfactory. In this contribution we present a number of techniques which allow to determine much more efficient upper bounds for stop-loss premiums. Like in Deelstra et al. (2004) and Vanmaele et al. (2004b), we use on one hand the method of conditioning as in Curran (1994) and in Rogers and Shi (1995), and on the other hand the upper and lower bounds for stop-loss premiums of sums of dependent random variables as derived in Dhaene et al. (2002a, 2002b). We provide a number of numerical illustrations which reveal a significant improvement compared to the traditional comonotonic approximations.

We show how to apply our results to the case of sums of lognormal distributed random variables. 
Such sums are widely encountered in practice, both in actuarial science and in finance. Typical examples are present values of future cash-flows with stochastic (Gaussian) interest rates (see Dhaene et al. (2002b), Asian options (see e.g. Simon et al. (2000) and Vanmaele et al. (2004b)) and basket options (see Deelstra et al. (2004) and Vanmaele et al. (2004a)). In the application section we show how to adapt the bounds to the case of a single life annuity (cash flows with a stochastic time horizon) and to the case of a diversified portfolio of life annuities (cash flows with a deterministic time horizon).

The paper is organized as follows. We recapitulate the theoretical results of Dhaene et al. (2002a) in Section 2. In Section 3 we apply the results of Rogers and Shi (1995) to get an alternative upper bound for stop-loss premiums. Section 4 explains how these upper bounds can be improved by decomposing stop-loss premiums. The application to the lognormal case and the generalization to sums with a stochastic time horizon are presented in Section 5. Section 6 contains some numerical illustrations for the case of life annuities. Finally in Section 7 we insert some concluding remarks.

\section{Some theoretical results}

In this section, we recall from Dhaene et al. (2002a) and the references therein the procedures for obtaining the lower and upper bounds for stop-loss premiums of sums $\mathbb{S}$ of dependent random variables by using the notion of comonotonicity. A random vector $\left(X_{1}^{c}, \ldots, X_{n}^{c}\right)$ is comonotonic if each two possible outcomes $\left(x_{1}, \ldots, x_{n}\right)$ and $\left(y_{1}, \ldots, y_{n}\right)$ of $\left(X_{1}^{c}, \ldots, X_{n}^{c}\right)$ are ordered componentwise.

In both financial and actuarial context one encounters quite often random variables of the type $\mathbb{S}=\sum_{i=1}^{n} X_{i}$ where the terms $X_{i}$ are not mutually independent, but the multivariate distribution function of the random vector $\underline{X}=\left(X_{1}, X_{2}, \ldots, X_{n}\right)$ is not completely specified because one only knows the marginal distribution functions of the random variables $X_{i}$. In such cases, to be able to make decisions it may be helpful to find the dependence structure for the random vector $\left(X_{1}, \ldots, X_{n}\right)$ producing the least favourable aggregate claims $\mathbb{S}$ with given marginals. Therefore, given the marginal distributions of the terms in a random variable $\mathbb{S}=\sum_{i=1}^{n} X_{i}$, we shall look for the joint distribution with a smaller resp. larger sum, in the convex order $\left(\leq_{c x}\right)$ sense, which means that $\mathbb{S}_{1} \leq_{c x} \mathbb{S}_{2} \Leftrightarrow \mathrm{E}\left[\mathbb{S}_{1}\right]=\mathrm{E}\left[\mathbb{S}_{2}\right]$ and $\mathrm{E}\left[\left(\mathbb{S}_{1}-d\right)_{+}\right] \leq \mathrm{E}\left[\left(\mathbb{S}_{2}-d\right)_{+}\right]$for all $d \in \mathbb{R}$. In short, the sum $\mathbb{S}$ is bounded below and above in convex order by the following sums which will be defined in the subsequent sections:

$$
\mathbb{S}^{\ell} \leq_{c x} \mathbb{S} \leq_{c x} \mathbb{S}^{u} \leq_{c x} \mathbb{S}^{c}
$$

which implies by definition of convex order that

$$
\mathrm{E}\left[\left(\mathbb{S}^{\ell}-d\right)_{+}\right] \leq \mathrm{E}\left[(\mathbb{S}-d)_{+}\right] \leq \mathrm{E}\left[\left(\mathbb{S}^{u}-d\right)_{+}\right] \leq \mathrm{E}\left[\left(\mathbb{S}^{c}-d\right)_{+}\right]
$$

for all $d$ in $\mathbb{R}$, while $\mathrm{E}\left[\mathbb{S}^{\ell}\right]=\mathrm{E}[\mathbb{S}]=\mathrm{E}\left[\mathbb{S}^{u}\right]=\mathrm{E}\left[\mathbb{S}^{c}\right]$ and $\operatorname{Var}\left[\mathbb{S}^{\ell}\right] \leq \operatorname{Var}[\mathbb{S}] \leq \operatorname{Var}\left[\mathbb{S}^{u}\right] \leq \operatorname{Var}\left[\mathbb{S}^{c}\right]$. We recall that $(x)_{+}=\max \{x, 0\}$. 


\subsection{Comonotonic upper bound}

As proven in Dhaene et al. (2002a), the convex-largest sum of the components of a random vector with given marginals is obtained by the comonotonic sum $\mathbb{S}^{c}=X_{1}^{c}+X_{2}^{c}+\cdots+X_{n}^{c}$ with

$$
\mathbb{S}^{c} \stackrel{d}{=} \sum_{i=1}^{n} F_{X_{i}}^{-1}(U)
$$

where " $\stackrel{d}{=}$ means equality in distribution and where $U$ denotes in the following a Uniform $(0,1)$ random variable. The usual inverse of a distribution function, which is the non-decreasing and left-continuous function $F_{X}^{-1}(p)$, is defined by

$$
F_{X}^{-1}(p)=\inf \left\{x \in \mathbb{R} \mid F_{X}(x) \geq p\right\}, \quad p \in[0,1],
$$

with $\inf \emptyset=+\infty$ by convention.

Kaas et al. (2000) have proved that the inverse distribution function of a sum of comonotonic random variables is simply the sum of the inverse distribution functions of the marginal distributions. Moreover, in case of strictly increasing and continuous marginals, the cumulative distribution function (cdf) $F_{\mathbb{S}^{c}}(x)$ is uniquely determined by

$$
F_{\mathbb{S}^{c}}^{-1}\left(F_{\mathbb{S} c}(x)\right)=\sum_{i=1}^{n} F_{X_{i}}^{-1}\left(F_{\mathbb{S} c}(x)\right)=x, \quad F_{\mathbb{S}^{c}}^{-1}(0)<x<F_{\mathbb{S}^{c}}^{-1}(1) .
$$

Hereafter we restrict ourselves to this case of strictly increasing and continuous marginals.

In the following theorem Dhaene et al. (2002) have proved that the stop-loss premiums of a sum of comonotonic random variables can easily be obtained from the stop-loss premiums of the terms.

Theorem 1 The stop-loss premium, denoted by $\pi^{c u b}(\mathbb{S}, d)$, of the sum $\mathbb{S}^{c}$ of the components of the comonotonic random vector $\left(X_{1}^{c}, X_{2}^{c}, \ldots, X_{n}^{c}\right)$ at retention $d$ is given by

$$
\pi^{c u b}(\mathbb{S}, d)=\sum_{i=1}^{n} \mathrm{E}\left[\left(X_{i}-F_{X_{i}}^{-1}\left(F_{\mathbb{S}^{c}}(d)\right)\right)_{+}\right], \quad\left(F_{\mathbb{S}^{c}}^{-1}(0)<d<F_{\mathbb{S}^{c}}^{-1}(1)\right) .
$$

If the only information available concerning the multivariate distribution function of the random vector $\left(X_{1}, \ldots, X_{n}\right)$ are the marginal distribution functions of the $X_{i}$, then the distribution function of $\mathbb{S}^{c}=F_{X_{1}}^{-1}(U)+F_{X_{2}}^{-1}(U)+\cdots+F_{X_{n}}^{-1}(U)$ is a prudent choice for approximating the unknown distribution function of $\mathbb{S}=X_{1}+\cdots+X_{n}$. It is a supremum in terms of convex order. It is the best upper bound that can be derived under the given conditions.

\subsection{Improved comonotonic upper bound}

Let us now assume that we have some additional information available concerning the stochastic nature of $\left(X_{1}, \ldots, X_{n}\right)$. More precisely, we assume that there exists some random variable $\Lambda$ with a given distribution function, such that we know the conditional cumulative distribution 
functions, given $\Lambda=\lambda$, of the random variables $X_{i}$, for all possible values of $\lambda$. In fact, Kaas et al. (2000) define the improved comonotonic upper bound $\mathbb{S}^{u}$ as

$$
\mathbb{S}^{u}=F_{X_{1} \mid \Lambda}^{-1}(U)+F_{X_{2} \mid \Lambda}^{-1}(U)+\cdots+F_{X_{n} \mid \Lambda}^{-1}(U),
$$

where $F_{X_{i} \mid \Lambda}^{-1}(U)$ is the notation for the random variable $f_{i}(U, \Lambda)$, with the function $f_{i}$ defined by $f_{i}(u, \lambda)=F_{X_{i} \mid \Lambda=\lambda}^{-1}(u)$. In order to obtain the distribution function of $\mathbb{S}^{u}$, observe that given the event $\Lambda=\lambda$, the random variable $\mathbb{S}^{u}$ is a sum of comonotonic random variables. If the marginal cdfs $F_{X_{i} \mid \Lambda=\lambda}$ are strictly increasing and continuous, then $F_{\mathbb{S} u \mid \Lambda=\lambda}(x)$ is a solution to

$$
\sum_{i=1}^{n} F_{X_{i} \mid \Lambda=\lambda}^{-1}\left(F_{\mathbb{S}^{u} \mid \Lambda=\lambda}(x)\right)=x, \quad x \in\left(F_{\mathbb{S}^{u} \mid \Lambda=\lambda}^{-1}(0), F_{\mathbb{S}^{u} \mid \Lambda=\lambda}^{-1}(1)\right),
$$

and the cdf of $\mathbb{S}^{u}$ then follows from

$$
F_{\mathbb{S} u}(x)=\int_{-\infty}^{+\infty} F_{\mathbb{S} u \mid \Lambda=\lambda}(x) d F_{\Lambda}(\lambda)
$$

In this case, we also find that for any $d \in\left(F_{\mathbb{S} u \mid \Lambda=\lambda}^{-1}(0), F_{\mathbb{S}^{u} \mid \Lambda=\lambda}^{-1}(1)\right)$ :

$$
\mathrm{E}\left[\left(\mathbb{S}^{u}-d\right)_{+} \mid \Lambda=\lambda\right]=\sum_{i=1}^{n} \mathrm{E}\left[\left(X_{i}-F_{X_{i} \mid \Lambda=\lambda}^{-1}\left(F_{\mathbb{S}^{u} \mid \Lambda=\lambda}(d)\right)\right)_{+} \mid \Lambda=\lambda\right],
$$

from which the stop-loss premium at retention $d$ of $\mathbb{S}^{u}$, denoted by $\pi^{i c u b}(\mathbb{S}, d, \Lambda)$, can be determined by integration with respect to $\lambda$ over the real line.

\section{$2.3 \quad$ Lower bound}

Let $\underline{X}=\left(X_{1}, \ldots, X_{n}\right)$ be a random vector with given marginal cdfs $F_{X_{1}}, F_{X_{2}}, \ldots, F_{X_{n}}$. Assume again that there exists some random variable $\Lambda$ with a given distribution function, such that we know the conditional distribution, given $\Lambda=\lambda$, of the random variables $X_{i}$, for all possible values of $\lambda$. We recall from Kaas et al. (2000) that a lower bound, in the sense of convex order, for $\mathbb{S}=X_{1}+X_{2}+\cdots+X_{n}$ is

$$
\mathbb{S}^{\ell}=\mathrm{E}[\mathbb{S} \mid \Lambda]
$$

This idea can also be found in Rogers and Shi (1995) for the continuous case.

Let us further assume that the random variable $\Lambda$ is such that all $\mathrm{E}\left[X_{i} \mid \Lambda\right]$ are non-decreasing and continuous functions of $\Lambda$, then $\mathbb{S}^{\ell}$ is a comonotonic sum. When in addition the cdfs of the random variables $\mathrm{E}\left[X_{i} \mid \Lambda\right]$ are strictly increasing and continuous, then the cdf of $\mathbb{S}^{\ell}$ is also strictly increasing and continuous, and we get analogously to (1) for all $x \in\left(F_{\mathbb{S} \ell}^{-1}(0), F_{\mathbb{S} \ell}^{-1}(1)\right)$,

$$
\sum_{i=1}^{n} F_{\mathrm{E}\left[X_{i} \mid \Lambda\right]}^{-1}\left(F_{\mathbb{S}^{\ell}}(x)\right)=x \quad \Leftrightarrow \quad \sum_{i=1}^{n} \mathrm{E}\left[X_{i} \mid \Lambda=F_{\Lambda}^{-1}\left(F_{\mathbb{S} \ell}(x)\right)\right]=x,
$$


which unambiguously determines the cdf of the convex order lower bound $\mathbb{S}^{\ell}$ for $\mathbb{S}$. In order to derive the above equivalence, we used the fact that for a non-decreasing continuous function $g$, we have

$$
F_{g(X)}^{-1}(p)=g\left(F_{X}^{-1}(p)\right), \quad p \in(0,1) .
$$

Invoking Theorem 1 , the stop-loss premium $\pi^{\ell b}(\mathbb{S}, d, \Lambda)$ of $\mathbb{S}^{\ell}$ can be computed as:

$$
\pi^{\ell b}(\mathbb{S}, d, \Lambda)=\sum_{i=1}^{n} \mathrm{E}\left[\left(\mathrm{E}\left[X_{i} \mid \Lambda\right]-\mathrm{E}\left[X_{i} \mid \Lambda=F_{\Lambda}^{-1}\left(F_{\mathbb{S}^{\ell}}(d)\right)\right]\right)_{+}\right]
$$

which holds for all retentions $d \in\left(F_{\mathbb{S} \ell}^{-1}(0), F_{\mathbb{S} \ell}^{-1}(1)\right)$.

So far, we considered the case that all $\mathrm{E}\left[X_{i} \mid \Lambda\right]$ are non-decreasing functions of $\Lambda$. The case where all $\mathrm{E}\left[X_{i} \mid \Lambda\right]$ are non-increasing and continuous functions of $\Lambda$ also leads to a comonotonic vector $\left(\mathrm{E}\left[X_{1} \mid \Lambda\right], \mathrm{E}\left[X_{2} \mid \Lambda\right], \ldots, \mathrm{E}\left[X_{n} \mid \Lambda\right]\right)$, and can be treated in a similar way but will not be dealt with in this paper.

In case the cdfs of the random variables $\mathrm{E}\left[X_{i} \mid \Lambda\right]$ are not continuous nor strictly increasing or decreasing functions of $\Lambda$, then the stop-loss premiums of $\mathbb{S}^{\ell}$, which is not comonotonic anymore, can be determined as follows :

$$
\pi^{\ell b}(\mathbb{S}, d, \Lambda)=\int_{-\infty}^{+\infty}\left(\sum_{i=1}^{n} \mathrm{E}\left[X_{i} \mid \Lambda=\lambda\right]-d\right)_{+} d F_{\Lambda}(\lambda)
$$

\section{Upper bounds based on lower bound plus error term}

Following the ideas of Rogers and Shi (1995), we derive an upper bound based on the lower bound. Indeed, applying the following general inequality for any random variable $Y$ and $Z$ from Rogers and Shi (1995):

$$
0 \leq \mathrm{E}\left[\mathrm{E}\left[Y_{+} \mid Z\right]-\mathrm{E}[Y \mid Z]_{+}\right] \leq \frac{1}{2} \mathrm{E}[\sqrt{\operatorname{Var}(Y \mid Z)}]
$$

to the case of $Y$ being $\mathbb{S}-d$ and $Z$ being our conditioning variable $\Lambda$, we obtain an error bound

$$
0 \leq \mathrm{E}\left[\mathrm{E}\left[(\mathbb{S}-d)_{+} \mid \Lambda\right]-\left(\mathbb{S}^{\ell}-d\right)_{+}\right] \leq \frac{1}{2} \mathrm{E}[\sqrt{\operatorname{Var}(\mathbb{S} \mid \Lambda)}]
$$

which is only useful if the retention $d$ is strictly positive.

Consequently, we find as upper bound for the stop-loss premium of $\mathbb{S}$

$$
\pi(\mathbb{S}, d) \leq \pi^{e u b}(\mathbb{S}, d, \Lambda),
$$

with $\pi^{e u b}(\mathbb{S}, d, \Lambda)$ given by

$$
\pi^{e u b}(\mathbb{S}, d, \Lambda)=\pi^{\ell b}(\mathbb{S}, d, \Lambda)+\frac{1}{2} \mathrm{E}[\sqrt{\operatorname{Var}(\mathbb{S} \mid \Lambda)}] .
$$


The second term on the right hand side takes the form

$$
\begin{aligned}
\mathrm{E}[\sqrt{\operatorname{Var}(\mathbb{S} \mid \Lambda)}] & =\mathrm{E}\left[\left(\mathrm{E}\left[\mathbb{S}^{2} \mid \Lambda\right]-(\mathrm{E}[\mathbb{S} \mid \Lambda])^{2}\right)^{1 / 2}\right] \\
& =\mathrm{E}\left[\left(\sum_{i=1}^{n} \sum_{j=1}^{n} \mathrm{E}\left[X_{i} X_{j} \mid \Lambda\right]-\left(\mathbb{S}^{\ell}\right)^{2}\right)^{1 / 2}\right]
\end{aligned}
$$

and once the distributions of $X_{i}$ and $\Lambda$ are specified and known, it can be written out more explicitly.

\section{Bounds by conditioning through decomposition of the stop- loss premium}

\subsection{Decomposition of the stop-loss premium}

In this section we show how to improve the bounds introduced in Sections 2-3. By conditioning $\mathbb{S}$ on some random variable $\Lambda$, the stop-loss premium can be decomposed in two parts, one of which can either be computed exactly or by using numerical integration, depending on the distribution of the underlying random variable. For the remaining part we first derive a lower and an upper bound based on comonotonic risks, and another upper bound equal to that lower bound plus an error term. This idea of decomposition goes back at least to Curran (1994).

By the tower property for conditional expectations the stop-loss premium $\pi(\mathbb{S}, d)$ with $\mathbb{S}=\sum_{i=1}^{n} X_{i}$ equals

$$
\mathrm{E}\left[\mathrm{E}\left[(\mathbb{S}-d)_{+} \mid \Lambda\right]\right]
$$

for some conditioning variable $\Lambda$ with $\operatorname{cdf} F_{\Lambda}$.

If in addition there exists a $d_{\Lambda}$ such that $\Lambda \geq d_{\Lambda}$ implies that $\mathbb{S} \geq d$ or such that $\Lambda \leq d_{\Lambda}$ implies that $\mathbb{S} \geq d$, it holds that

$$
\mathrm{E}\left[(\mathbb{S}-d)_{+} \mid \Lambda\right]=\mathrm{E}[\mathbb{S}-d \mid \Lambda] \stackrel{(3)}{=}\left(\mathbb{S}^{\ell}-d\right)_{+} .
$$

Note that in practical applications the existence of such a $d_{\Lambda}$ depends on the actual form of $\mathbb{S}$ and $\Lambda$.

We now concentrate upon the decomposition in the first case, the second case can be treated in a similar way with the appropriate integration bounds:

$$
\begin{aligned}
\pi(\mathbb{S}, d) & =\int_{-\infty}^{d_{\Lambda}} \mathrm{E}\left[(\mathbb{S}-d)_{+} \mid \Lambda=\lambda\right] d F_{\Lambda}(\lambda)+\int_{d_{\Lambda}}^{+\infty} \mathrm{E}[\mathbb{S}-d \mid \Lambda=\lambda] d F_{\Lambda}(\lambda) \\
& \stackrel{\text { not }}{=} I_{1}+I_{2} .
\end{aligned}
$$

The second integral can further be simplified to

$$
I_{2}=\int_{d_{\Lambda}}^{+\infty} \sum_{i=1}^{n} \mathrm{E}\left[X_{i} \mid \Lambda=\lambda\right] d F_{\Lambda}(\lambda)-d\left(1-F_{\Lambda}\left(d_{\Lambda}\right)\right),
$$


and can be written out explicitly if the bivariate distribution of $\left(X_{i}, \Lambda\right)$ is known for all $i$.

Deriving bounds for the first part $I_{1}$ in decomposition (11) and adding up to the exact part (12) gives us the bounds for the stop-loss premium.

\subsection{Lower bound}

By means of Jensen's inequality, the first integral $I_{1}$ of $(11)$ can be bounded below:

$$
I_{1} \geq \int_{-\infty}^{d_{\Lambda}}(\mathrm{E}[\mathbb{S} \mid \Lambda=\lambda]-d)_{+} d F_{\Lambda}(\lambda)=\int_{-\infty}^{d_{\Lambda}}\left(\sum_{i=1}^{n} \mathrm{E}\left[X_{i} \mid \Lambda=\lambda\right]-d\right)_{+} d F_{\Lambda}(\lambda) .
$$

By adding the exact part (12) and introducing notation (3), we end up with the inequality of Section 2.3:

$$
\pi(\mathbb{S}, d) \geq \pi^{\ell b}(\mathbb{S}, d, \Lambda)
$$

When $\mathbb{S}^{\ell}$ is a sum of $n$ comonotonic risks we can apply (5) which holds even when we do not know or find a $d_{\Lambda}$.

When $\mathbb{S}^{\ell}$ is not comonotonic we use the decomposition

$\pi^{\ell b}(\mathbb{S}, d, \Lambda)=\int_{-\infty}^{d_{\Lambda}}\left(\sum_{i=1}^{n} \mathrm{E}\left[X_{i} \mid \Lambda=\lambda\right]-d\right)_{+} d F_{\Lambda}(\lambda)+\int_{d_{\Lambda}}^{+\infty} \sum_{i=1}^{n} \mathrm{E}\left[X_{i} \mid \Lambda=\lambda\right] d F_{\Lambda}(\lambda)-d\left(1-F_{\Lambda}\left(d_{\Lambda}\right)\right)$.

\subsection{Upper bound based on lower bound}

In this section we improve the bound (8) by applying (6) to (13):

$$
\begin{aligned}
0 & \leq \mathrm{E}\left[\mathrm{E}\left[(\mathbb{S}-d)_{+} \mid \Lambda\right]-\left(\mathbb{S}^{\ell}-d\right)_{+}\right] \\
& =\int_{-\infty}^{d_{\Lambda}}\left(\mathrm{E}\left[(\mathbb{S}-d)_{+} \mid \Lambda=\lambda\right]-(\mathrm{E}[\mathbb{S} \mid \Lambda=\lambda]-d)_{+}\right) d F_{\Lambda}(\lambda) \\
& \leq \frac{1}{2} \int_{-\infty}^{d_{\Lambda}}(\operatorname{Var}(\mathbb{S} \mid \Lambda=\lambda))^{\frac{1}{2}} d F_{\Lambda}(\lambda) \\
& \leq \frac{1}{2}\left(\mathrm{E}\left[\operatorname{Var}(\mathbb{S} \mid \Lambda) 1_{\left\{\Lambda<d_{\Lambda}\right\}}\right]\right)^{\frac{1}{2}}\left(\mathrm{E}\left[1_{\left\{\Lambda<d_{\Lambda}\right\}}\right]\right)^{\frac{1}{2}} \stackrel{\text { not }}{=} \varepsilon\left(d_{\Lambda}\right),
\end{aligned}
$$

where Hölder's inequality has been applied in the last inequality and where $1_{\left\{\Lambda<d_{\Lambda}\right\}}$ is the indicator function, i.e. $1_{\{c\}}=1$ if the condition $c$ is true and $1_{\{c\}}=0$ if it is not. We will denote this upper bound by $\pi^{d e u b}(\mathbb{S}, d, \Lambda)$. So we have that

$$
\pi^{d e u b}(\mathbb{S}, d, \Lambda)=\pi^{\ell b}(\mathbb{S}, d, \Lambda)+\varepsilon\left(d_{\Lambda}\right)
$$

We remark that the error bound (7), and hence also the upper bound $\pi^{e u b}(\mathbb{S}, d, \Lambda)$, is independent of $d_{\Lambda}$ and corresponds to the limiting case of (14) where $d_{\Lambda}$ equals infinity. Obviously, the error bound (14) improves the error bound (7). In practical applications, the additional error introduced by Hölders inequality turns out to be much smaller than the difference $\frac{1}{2} \mathrm{E}[\sqrt{\operatorname{Var}(\mathbb{S} \mid \Lambda)}]-\varepsilon\left(d_{\Lambda}\right)$. 


\subsection{Partially exact/comonotonic upper bound}

We bound the first term $I_{1}$ of (11) above by replacing $\mathbb{S} \mid \Lambda=\lambda$ by its comonotonic upper bound $\mathbb{S}^{u}$ (in convex order sense):

$$
\int_{-\infty}^{d_{\Lambda}} \mathrm{E}\left[(\mathbb{S}-d)_{+} \mid \Lambda=\lambda\right] d F_{\Lambda}(\lambda) \leq \int_{-\infty}^{d_{\Lambda}} \mathrm{E}\left[\left(\mathbb{S}^{u}-d\right)_{+} \mid \Lambda=\lambda\right] d F_{\Lambda}(\lambda) .
$$

Adding (17) to the exact part (12) of the decomposition (11) results in the so-called partially exact/comonotonic upper bound for a stop-loss premium. We will use the notation $\pi^{\text {pecub }}(\mathbb{S}, d, \Lambda)$ to indicate this upper bound.

It is easily seen that

$$
\pi^{\text {pecub }}(\mathbb{S}, d, \Lambda) \leq \pi^{i c u b}(\mathbb{S}, d, \Lambda),
$$

while for two distinct conditioning variables $\Lambda_{1}$ and $\Lambda_{2}$ it does not necessarily holds that

$$
\pi^{p e c u b}\left(\mathbb{S}, d, \Lambda_{1}\right) \leq \pi^{i c u b}\left(\mathbb{S}, d, \Lambda_{2}\right)
$$

\section{Case of sum of lognormal random variables}

In this section we further develop the expressions for the lower and upper bounds when the random variables $X_{i}$ in the sum $\mathbb{S}$ are lognormal.

We assume that $X_{i}=\alpha_{i} e^{Z_{i}}$ with $Z_{i} \sim N\left(\mathrm{E}\left[Z_{i}\right], \sigma_{Z_{i}}\right)$ and $\alpha_{i} \in \mathbb{R}$, i.e.

$$
\mathbb{S}=\sum_{i=1}^{n} X_{i}=\sum_{i=1}^{n} \alpha_{i} e^{Z_{i}}
$$

In this case the stop-loss premium with some retention $d_{i}$, namely $\pi\left(X_{i}, d_{i}\right)$, is well-known from the following lemma.

Lemma 1 Let $X$ be a lognormal random variable of the form $\alpha e^{Z}$ with $Z \sim N\left(\mathrm{E}[Z], \sigma_{Z}\right)$ and $\alpha \in \mathbb{R}$. Then the stop-loss premium with retention $d$ equals for $\alpha d>0$

$$
\pi(X, d)=\operatorname{sign}(\alpha) e^{\mu+\frac{\sigma^{2}}{2}} \Phi\left(\operatorname{sign}(\alpha) b_{1}\right)-d \Phi\left(\operatorname{sign}(\alpha) b_{2}\right)
$$

where

$$
\begin{aligned}
\mu & =\ln |\alpha|+\mathrm{E}[Z] & \sigma & =\sigma_{Z} \\
b_{1} & =\frac{\mu+\sigma^{2}-\ln |d|}{\sigma} & b_{2} & =b_{1}-\sigma
\end{aligned}
$$

and where $\Phi$ stands for the cdf of a standard normal random variable. The cases $\alpha d<0$ are trivial.

We now consider a normally distributed random variable $\Lambda$. The following results are analogous to Theorem 1 in Dhaene et al. (2002b). 
Theorem 2 Let $\mathbb{S}$ be given by (18) and consider a normally distributed random variable $\Lambda$ which is such that $\left(Z_{i}, \Lambda\right)$ is bivariate normally distributed for all $i$. Then the distributions of the lower bound $\mathbb{S}^{\ell}$, the improved comonotonic upper bound $\mathbb{S}^{u}$ and the comonotonic upper bound $\mathbb{S}^{c}$ are given by

$$
\begin{aligned}
\mathbb{S}^{\ell} & =\sum_{i=1}^{n} \alpha_{i} e^{\mathrm{E}\left[Z_{i}\right]+r_{i} \sigma_{Z_{i}} \Phi^{-1}(V)+\frac{1}{2}\left(1-r_{i}^{2}\right) \sigma_{Z_{i}}^{2}}, \\
\mathbb{S}^{u} & =\sum_{i=1}^{n} \alpha_{i} e^{\mathrm{E}\left[Z_{i}\right]+r_{i} \sigma_{Z_{i}} \Phi^{-1}(V)+\operatorname{sign}\left(\alpha_{i}\right) \sqrt{1-r_{i}^{2}} \sigma_{Z_{i}} \Phi^{-1}(U)} \\
\mathbb{S}^{c} & =\sum_{i=1}^{n} \alpha_{i} e^{\mathrm{E}\left[Z_{i}\right]+\operatorname{sign}\left(\alpha_{i}\right) \sigma_{Z_{i}} \Phi^{-1}(U)}
\end{aligned}
$$

where $U$ and $V=\Phi\left(\frac{\Lambda-\mathrm{E}[\Lambda]}{\sigma_{\Lambda}}\right)$ are mutually independent uniform $(0,1)$ random variables, $\Phi$ is the cdf of the $N(0,1)$ distribution and $r_{i}, i=1, \ldots, n$, are correlations defined by

$$
r_{i}=r\left(Z_{i}, \Lambda\right)=\frac{\operatorname{Cov}\left[Z_{i}, \Lambda\right]}{\sigma_{Z_{i}} \sigma_{\Lambda}}
$$

When for all $i \operatorname{sign}\left(\alpha_{i}\right)=\operatorname{sign}\left(r_{i}\right)$ or for all $i \operatorname{sign}\left(\alpha_{i}\right)=-\operatorname{sign}\left(r_{i}\right)$ for $r_{i} \neq 0$ then $\mathbb{S}^{\ell}$ is comonotonic.

\subsection{Comonotonic upper bound}

Since the cdfs $F_{X_{i}}$ are strictly increasing and continuous, it follows from (1) and (23) that for $x \in\left(F_{\mathbb{S c}}^{-1}(0), F_{\mathbb{S}^{c}}^{-1}(1)\right)$, the cdf of the comonotonic sum $F_{\mathbb{S c}}(x)$ can be found by solving

$$
\sum_{i=1}^{n} \alpha_{i} e^{\mathrm{E}\left[Z_{i}\right]+\operatorname{sign}\left(\alpha_{i}\right) \sigma_{Z_{i}} \Phi^{-1}\left(F_{\mathbb{S}}(x)\right)}=x .
$$

Combination of Theorem 1 and Lemma 1 yields the following expression for the stop-loss premium of $\mathbb{S}^{c}$ at retention $d$ with $F_{\mathbb{S}^{c}}^{-1}(0)<d<F_{\mathbb{S}^{c}}^{-1}(1)$ :

$$
\pi^{c u b}(\mathbb{S}, d)=\sum_{i=1}^{n} \alpha_{i} e^{\mathrm{E}\left[Z_{i}\right]+\frac{\sigma_{Z_{i}}^{2}}{2}} \Phi\left[\operatorname{sign}\left(\alpha_{i}\right) \sigma_{Z_{i}}-\Phi^{-1}\left(F_{\mathbb{S} c}(d)\right)\right]-d\left(1-F_{\mathbb{S}^{c}}(d)\right) .
$$

\subsection{Improved comonotonic upper bound}

We now determine the cdf of $\mathbb{S}^{u}$ and the stop-loss premium $\pi^{i c u b}(\mathbb{S}, d, \Lambda)$, where we condition on a normally distributed random variable $\Lambda$ or equivalently on the uniform $(0,1)$ random variable introduced in Theorem 2:

$$
V=\Phi\left(\frac{\Lambda-\mathrm{E}[\Lambda]}{\sigma_{\Lambda}}\right)
$$

The conditional probability $F_{\mathbb{S}^{u} \mid V=v}(x)$ also denoted by $F_{\mathbb{S} u}(x \mid V=v)$, is the cdf of a sum of $n$ comonotonic random variables and follows for $F_{\mathbb{S}^{u} \mid V=v}^{-1}(0)<x<F_{\mathbb{S}^{u} \mid V=v}^{-1}(1)$, according to (2) 
and (22), implicitly from:

$$
\sum_{i=1}^{n} \alpha_{i} e^{\mathrm{E}\left[Z_{i}\right]+r_{i} \sigma_{Z_{i}} \Phi^{-1}(v)+\operatorname{sign}\left(\alpha_{i}\right) \sqrt{1-r_{i}^{2}} \sigma_{Z_{i}} \Phi^{-1}\left(F_{\mathbb{S} u}(x \mid V=v)\right)}=x .
$$

The cdf of $\mathbb{S}^{u}$ is then given by

$$
F_{\mathbb{S}^{u}}(x)=\int_{0}^{1} F_{\mathbb{S}^{u} \mid V=v}(x) d v .
$$

We now look for an expression for the stop-loss premium at retention $d$ with $F_{\mathbb{S}^{u} \mid V=v}^{-1}(0)<d<$ $F_{\mathbb{S}^{u} \mid V=v}^{-1}(1)$ for $\mathbb{S}^{u}:$

$$
\pi^{i c u b}(\mathbb{S}, d, \Lambda)=\int_{0}^{1} \mathrm{E}\left[\left(\mathbb{S}^{u}-d\right)_{+} \mid V=v\right] d v=\sum_{i=1}^{n} \int_{0}^{1} \mathrm{E}\left[\left(F_{X_{i} \mid \Lambda}^{-1}(U \mid V=v)-d_{i}\right)_{+}\right] d v
$$

with $d_{i}=F_{X_{i} \mid \Lambda}^{-1}\left(F_{\mathbb{S} u}(d \mid V=v) \mid V=v\right)$ and with $U$ a random variable which is uniformly distributed on $(0,1)$. Since $\operatorname{sign}\left(\alpha_{i}\right) F_{X_{i} \mid \Lambda}^{-1}(U \mid V=v)$ follows a lognormal distribution with mean and standard deviation:

$$
\mu_{v}(i)=\ln \left|\alpha_{i}\right|+\mathrm{E}\left[Z_{i}\right]+r_{i} \sigma_{Z_{i}} \Phi^{-1}(v), \quad \sigma_{v}(i)=\sqrt{1-r_{i}^{2}} \sigma_{Z_{i}},
$$

one obtains that

$$
d_{i}=\alpha_{i} \exp \left[\mathrm{E}\left[Z_{i}\right]+r_{i} \sigma_{Z_{i}} \Phi^{-1}(v)+\operatorname{sign}\left(\alpha_{i}\right) \sqrt{1-r_{i}^{2}} \sigma_{Z_{i}} \Phi^{-1}\left(F_{\mathbb{S}^{u} \mid V=v}(d)\right)\right] .
$$

The well-known formula (19) then yields

$$
\mathrm{E}\left[\left(\mathbb{S}^{u}-d\right)_{+} \mid V=v\right]=\sum_{i=1}^{n}\left[\operatorname{sign}\left(\alpha_{i}\right) e^{\mu_{v}(i)+\frac{\sigma_{v}^{2}(i)}{2}} \Phi\left(\operatorname{sign}\left(\alpha_{i}\right) b_{i, 1}\right)-d_{i} \Phi\left(\operatorname{sign}\left(\alpha_{i}\right) b_{i, 2}\right)\right],
$$

with, according to $(20)$,

$$
b_{i, 1}=\frac{\mu_{v}(i)+\sigma_{v}^{2}(i)-\ln \left|d_{i}\right|}{\sigma_{v}(i)}, \quad b_{i, 2}=b_{i, 1}-\sigma_{v}(i) .
$$

Substitution of the corresponding expressions and integration over the interval $[0,1]$ leads to the following result

$$
\begin{aligned}
\pi^{i c u b}(\mathbb{S}, d, \Lambda) & =\sum_{i=1}^{n} \alpha_{i} e^{\mathrm{E}\left[Z_{i}\right]+\frac{1}{2} \sigma_{Z_{i}}^{2}\left(1-r_{i}^{2}\right)} \int_{0}^{1} e^{r_{i} \sigma_{Z_{i}} \Phi^{-1}(v)} \times \\
& \times \Phi\left(\operatorname{sign}\left(\alpha_{i}\right) \sqrt{1-r_{i}^{2}} \sigma_{Z_{i}}-\Phi^{-1}\left(F_{\mathbb{S}^{u} \mid V=v}(d)\right)\right) d v-d\left(1-F_{\mathbb{S} u}(d)\right) .
\end{aligned}
$$




\subsection{Lower bound}

In this subsection, we study the case that, for all $i, \operatorname{sign}\left(\alpha_{i}\right)=\operatorname{sign}\left(r_{i}\right)$ when $r_{i} \neq 0$. For simplicity we take all $\alpha_{i} \geq 0$ and assume that the conditioning variable $\Lambda$ is normally distributed and has the right sign such that the correlation coefficients $r_{i}$ are all positive. These conditions ensure that $\mathbb{S}^{\ell}$ is the sum of $n$ comonotonic random variables. The case that, for all $i, \operatorname{sign}\left(\alpha_{i}\right)=$ $-\operatorname{sign}\left(r_{i}\right)$ when $r_{i} \neq 0$ can be dealt with in an analogous way.

Since by our assumptions $\mathrm{E}\left[X_{i} \mid \Lambda\right]$ is increasing, we can obtain $F_{\mathbb{S} \ell}(x)$ according to (4) and (21) from

$$
\sum_{i=1}^{n} \alpha_{i} e^{\mathrm{E}\left[Z_{i}\right]+r_{i} \sigma_{Z_{i}} \Phi^{-1}\left(F_{\mathbb{S} \ell}(x)\right)+\frac{1}{2}\left(1-r_{i}^{2}\right) \sigma_{Z_{i}}^{2}}=x .
$$

Moreover as $\mathbb{S}^{\ell}$ is the sum of $n$ lognormally distributed random variables, the stop-loss premium at retention $d(>0)$ can be expressed explicitly by invoking Theorem 1 and Lemma 1:

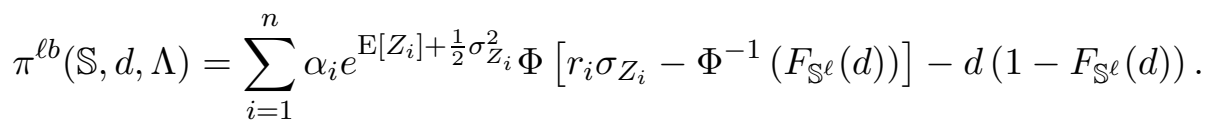

\subsection{Upper bound based on lower bound}

From (10) we obtain that

$$
\mathrm{E}[\sqrt{\operatorname{Var}(\mathbb{S} \mid \Lambda)}]=\int_{-\infty}^{+\infty}\left\{\sum_{i=1}^{n} \sum_{j=1}^{n} \mathrm{E}\left[X_{i} X_{j} \mid \Lambda=\lambda\right]-(\mathrm{E}[\mathbb{S} \mid \Lambda=\lambda])^{2}\right\}^{\frac{1}{2}} d F_{\Lambda}(\lambda) .
$$

Now consider the first term in the right hand side of (28). According to the properties of lognormally distributed random variables, the product of lognormals is again lognormal, and conditioning a lognormal variate on a normal variate yields a lognormally distributed variable.

We can proceed by denoting $Z_{i j}=Z_{i}+Z_{j}$ with $\mathrm{E}\left[Z_{i j}\right]=\mathrm{E}\left[Z_{i}\right]+\mathrm{E}\left[Z_{j}\right]$ and

$$
\sigma_{Z_{i j}}^{2}=\sigma_{Z_{i}}^{2}+\sigma_{Z_{j}}^{2}+2 \sigma_{Z_{i} Z_{j}}
$$

where $\sigma_{Z_{i} Z_{j}}$ stands for $\operatorname{Cov}\left(Z_{i}, Z_{j}\right)$. Note that

$$
\begin{aligned}
r_{i j} & =\frac{\operatorname{Cov}\left(Z_{i j}, \Lambda\right)}{\sigma_{Z_{i j}} \sigma_{\Lambda}} \\
& =\frac{\operatorname{Cov}\left(Z_{i}, \Lambda\right)}{\sigma_{Z_{i j}} \sigma_{\Lambda}}+\frac{\operatorname{Cov}\left(Z_{j}, \Lambda\right)}{\sigma_{Z_{i j}} \sigma_{\Lambda}} \\
& =\frac{\sigma_{Z_{i}}}{\sigma_{Z_{i j}}} r_{i}+\frac{\sigma_{Z_{j}}}{\sigma_{Z_{i j}}} r_{j} .
\end{aligned}
$$

Conditionally, given $\Lambda=\lambda$, the random variable $Z_{i j}$ is normally distributed with parameters $\mu(i j)=\mathrm{E}\left[Z_{i j}\right]+r_{i j} \frac{\sigma Z_{i j}}{\sigma_{\Lambda}}(\lambda-\mathrm{E}[\Lambda])$ and $\sigma^{2}(i j)=\left(1-r_{i j}^{2}\right) \sigma_{Z_{i j}}^{2}$. Hence, conditionally, given 
$\Lambda=\lambda$, the random variable $e^{Z_{i j}}$ is lognormally distributed with parameters $\mu(i j)$ and $\sigma^{2}(i j)$. As $\mathrm{E}\left[e^{Z_{i j}} \mid \Lambda=\lambda\right]=e^{\mu(i j)+\frac{1}{2} \sigma^{2}(i j)}$, we find

$$
\mathrm{E}\left[e^{Z_{i j}} \mid \Lambda\right]=e^{\mathrm{E}\left[Z_{i j}\right]+r_{i j} \sigma_{Z_{i j}} \Phi^{-1}(V)+\frac{1}{2}\left(1-r_{i j}^{2}\right) \sigma_{Z_{i j}}^{2}}
$$

where the random variable $V=\Phi\left(\frac{\Lambda-\mathrm{E}[\Lambda]}{\sigma_{\Lambda}}\right)$ is uniformly distributed on the interval $(0,1)$. Thus, the first term in (28) equals

$$
\sum_{i=1}^{n} \sum_{j=1}^{n} \mathrm{E}\left[X_{i} X_{j} \mid \Lambda\right]=\sum_{i=1}^{n} \sum_{j=1}^{n} \alpha_{i} \alpha_{j} \exp \left(\mathrm{E}\left[Z_{i j}\right]+r_{i j} \sigma_{Z_{i j}} \Phi^{-1}(V)+\frac{1}{2}\left(1-r_{i j}^{2}\right) \sigma_{Z_{i j}}^{2}\right)
$$

while the second term consists of (21). Hence (28) can be written out explicitly and by using (9) we have that the upper bound (8) is given by

$$
\begin{aligned}
\pi^{e u b}(\mathbb{S}, d, \Lambda)= & \sum_{i=1}^{n} \alpha_{i} e^{\mathrm{E}\left[Z_{i}\right]+\frac{1}{2} \sigma_{Z_{i}}^{2} \Phi\left[r_{i} \sigma_{Z_{i}}-\Phi^{-1}\left(F_{\mathbb{S}^{\ell}}(d)\right)\right]-d\left(1-F_{\mathbb{S}^{\ell}}(d)\right)+} \\
+\frac{1}{2} \int_{0}^{1} & \left\{\sum_{i=1}^{n} \sum_{j=1}^{n} \alpha_{i} \alpha_{j} e^{\mathrm{E}\left[Z_{i j}\right]+r_{i j} \sigma_{Z_{i j}} \Phi^{-1}(v)+\frac{1}{2}\left(1-r_{i j}^{2}\right) \sigma_{Z_{i j}}^{2}}-\right. \\
& \left.-\left(\sum_{i=1}^{n} \alpha_{i} e^{\mathrm{E}\left[Z_{i}\right]+r_{i} \sigma_{Z_{i}} \Phi^{-1}(v)+\frac{1}{2}\left(1-r_{i}^{2}\right) \sigma_{Z_{i}}^{2}}\right)^{2}\right\}^{\frac{1}{2}} d v
\end{aligned}
$$

\subsection{Bounds by conditioning through decomposition of stop-loss premium}

In this subsection we apply the theory of Section 4 to the sum of lognormal random variables (23). We only give here the analytical expressions for the two upper bounds $\pi^{\text {deub }}(\mathbb{S}, d, \Lambda)$ and $\pi^{\text {pecub }}(\mathbb{S}, d, \Lambda)$. For more details concerning the calculation of the bounds the reader is referred to Appendix A and B.

The following auxiliary result is needed in order to write out the bounds explicitely.

Lemma 2 For any constant $a \in \mathbb{R}$ and any normally distributed random variable $\Lambda$

$$
\int_{-\infty}^{d_{\Lambda}} e^{a \Phi^{-1}(v)} d F_{\Lambda}(\lambda)=e^{\frac{a^{2}}{2}} \Phi\left(d_{\Lambda}^{*}-a\right)
$$

where $d_{\Lambda}^{*}=\frac{d_{\Lambda}-\mathrm{E}[\Lambda]}{\sigma_{\Lambda}}, V=\Phi\left(\frac{\Lambda-\mathrm{E}[\Lambda]}{\sigma_{\Lambda}}\right)$ is uniformly distributed on the unit interval and thus, $\Phi^{-1}(V)=\frac{\Lambda-\mathrm{E}[\Lambda]}{\sigma_{\Lambda}}$ is a standard normal variable.

\subsubsection{Lower bound}

In view of the remark that the lower bound via the decomposition equals the lower bound without the decomposition, we refer for an expression for it in the lognormal and comonotonic case to Section 5.3. 


\subsubsection{Upper bound based on lower bound}

The upper bound (16) can be written out explicitly as follows

$$
\begin{aligned}
\pi^{\text {deub }}(\mathbb{S}, d, \Lambda)= & \sum_{i=1}^{n} \alpha_{i} e^{\mathrm{E}\left[Z_{i}\right]+\frac{1}{2} \sigma_{Z_{i}}^{2}} \Phi\left[r_{i} \sigma_{Z_{i}}-\Phi^{-1}\left(F_{\mathbb{S}_{\ell}}(d)\right)\right]-d\left(1-F_{\mathbb{S}^{\ell}}(d)\right)+ \\
+\frac{1}{2} \Phi\left(d_{\Lambda}^{*}\right)^{1 / 2} & \left\{\sum_{i=1}^{n} \sum_{j=1}^{n} \alpha_{i} \alpha_{j} e^{\mathrm{E}\left[Z_{i j}\right]+\frac{1}{2}\left(\sigma_{Z_{i}}^{2}+\sigma_{Z_{j}}^{2}\right)} \times\right. \\
& \left.\times \Phi\left(d_{\Lambda}^{*}-\left(r_{i} \sigma_{Z_{i}}+r_{j} \sigma_{Z_{j}}\right)\right)\left(e^{\sigma_{Z_{i} Z_{j}}}-e^{\sigma_{Z_{i}} \sigma_{Z_{j}} r_{i} r_{j}}\right)\right\}^{\frac{1}{2}} .
\end{aligned}
$$

\subsubsection{Partially exact/comonotonic upper bound}

The partially exact/comonotonic upper bound of subsection 4.4 is given by

$$
\begin{aligned}
\pi^{\text {pecub }}(\mathbb{S}, d, \Lambda)= & \sum_{i=1}^{n} \alpha_{i} e^{\mathrm{E}\left[Z_{i}\right]+\frac{1}{2} \sigma_{Z_{i}}^{2}\left(1-r_{i}^{2}\right)}\left\{e^{\frac{r_{i}^{2} \sigma_{Z_{i}}^{2}}{2}} \Phi\left(r_{i} \sigma_{Z_{i}}-d_{\Lambda}^{*}\right)+\int_{0}^{\Phi\left(d_{\Lambda}^{*}\right)} e^{r_{i} \sigma_{Z_{i}} \Phi^{-1}(v)} \times\right. \\
& \left.\times \Phi\left(\operatorname{sign}\left(\alpha_{i}\right) \sqrt{1-r_{i}^{2}} \sigma_{Z_{i}}-\Phi^{-1}\left(F_{\mathbb{S} u \mid V=v}(d)\right)\right) d v\right\}- \\
& -d\left(1-\int_{0}^{\Phi\left(d_{\Lambda}^{*}\right)} F_{\mathbb{S} u \mid V=v}(d) d v\right) .
\end{aligned}
$$

\subsection{Choice of the conditioning variable}

If $X \leq_{c x} Y$, and $X$ and $Y$ are not equal in distribution, then $\operatorname{Var}[X]<\operatorname{Var}[Y]$ must hold. An equality in variance would imply that $X \stackrel{d}{=} Y$. This shows that if we want to replace $\mathbb{S}$ by the less convex $\mathbb{S}^{\ell}$, the best approximations will occur when the variance of $\mathbb{S}^{\ell}$ is 'as close as possible' to the variance of $\mathbb{S}$. Hence we should choose $\Lambda$ such that goodness-of-fit expressed by the ratio $z=\frac{\operatorname{Var}\left(\mathbb{S}^{\ell}\right)}{\operatorname{Var}(\mathbb{S})}$ is as close as possible to 1 . Of course one can always use numerical procedures to optimize $z$ but this would outweigh one of the main features of the convex bounds, namely that the different relevant actuarial quantities (quantiles, tailvars, stop-loss premiums) can be easily obtained. Having a ready-to-use approximation that can be easily implemented and used by all kind of end-users is important from a business point of view.

We propose here three conditioning random variables. The first two are linear combinations of the random variables $Z_{i}$ :

$$
\Lambda=\sum_{i=1}^{n} \gamma_{i} Z_{i},
$$

for particular choices of the coefficients $\gamma_{i}$. 
Kaas, Dhaene and Goovaerts (2000) propose the following choice for the parameters $\gamma_{i}$ when computing the lower bound $\mathbb{S}^{\ell}$ :

$$
\gamma_{i}=\alpha_{i} e^{\mathrm{E}\left[Z_{i}\right]}, \quad i=1, \ldots, n .
$$

This choice makes $\Lambda$ a linear transformation of a first order approximation to $\mathbb{S}$. This can be seen from the following derivation:

$$
\begin{aligned}
\mathbb{S} & =\sum_{i=1}^{n} \alpha_{i} e^{\mathrm{E}\left[Z_{i}\right]+\left(Z_{i}-\mathrm{E}\left[Z_{i}\right]\right)} \approx \sum_{i=1}^{n} \alpha_{i} e^{\mathrm{E}\left[Z_{i}\right]}\left(1+Z_{i}-\mathrm{E}\left[Z_{i}\right]\right) \\
& \approx C+\sum_{i=1}^{n} \alpha_{i} e^{\mathrm{E}\left[Z_{i}\right]} Z_{i},
\end{aligned}
$$

where $C$ is the appropriate constant. Hence $\mathbb{S}^{\ell}$ will be "close" to $\mathbb{S}$, provided $\left(Z_{i}-\mathrm{E}\left(Z_{i}\right)\right)$ is sufficiently small, or equivalently, $\sigma_{Z_{i}}^{2}$ is sufficiently small. One intuitively expects that for this choice for $\Lambda, \mathrm{E}(\operatorname{Var}[\mathbb{S} \mid \Lambda])$ is "small" and since $\operatorname{Var}(\mathbb{S})=\mathrm{E}(\operatorname{Var}[\mathbb{S} \mid \Lambda])+\operatorname{Var}\left(\mathbb{S}^{\ell}\right)$ this exactly means that one expects the ratio $z=\frac{\operatorname{Var}\left(\mathbb{S}^{\ell}\right)}{\operatorname{Var}(\mathbb{S})}$ to tend to one.

A possible decomposition variable is in that case given by

$$
d_{\Lambda}=d-C=d-\sum_{i=1}^{n} \alpha_{i} e^{\mathrm{E}\left[Z_{i}\right]}\left(1-\mathrm{E}\left[Z_{i}\right]\right) .
$$

Using the property that $e^{x} \geq 1+x$ and (32), we have that $\Lambda \geq d_{\Lambda}$ implies that $\mathbb{S} \geq d$.

A second conditioning variable is proposed by Vanduffel, Hoedemakers and Dhaene (2004) for which the first order approximation of $\operatorname{Var}\left(\mathbb{S}^{\ell}\right)$ is maximized. They take in expression (31) for $\Lambda$ the parameters $\gamma_{i}$ equal to

$$
\gamma_{i}=\alpha_{i} e^{\mathrm{E}\left[Z_{i}\right]+\frac{1}{2} \sigma_{Z_{i}}^{2}}, \quad i=1, \ldots, n .
$$

For this 'maximal variance' conditioning variable a possible choice for $d_{\Lambda}$ is given by

$$
d_{\Lambda}=d-\sum_{i=1}^{n} \alpha_{i} e^{\mathrm{E}\left[Z_{i}\right]+\frac{1}{2} \sigma_{Z_{i}}^{2}}\left(1-\mathrm{E}\left[Z_{i}\right]-\frac{1}{2} \sigma_{Z_{i}}^{2}\right) .
$$

A third conditioning variable is based on the standardized logarithm of the geometric average $\mathbb{G}=\left(\prod_{i=1}^{n} \mathbb{S}\right)^{1 / n}$ as in Nielsen and Sandman (2002)

$$
\Lambda=\frac{\ln \mathbb{G}-\mathrm{E}[\ln \mathbb{G}]}{\sqrt{\operatorname{Var}(\ln \mathbb{G})}}=\frac{\sum_{i=1}^{n}\left(Z_{i}-\mathrm{E}\left[Z_{i}\right]\right)}{\sqrt{\operatorname{Var}\left(\sum_{i=1}^{n} Z_{i}\right)}} .
$$

Using the fact that the geometric average is not greater than the arithmetic average, a possible decomposition variable is here given by

$$
d_{\Lambda}=\frac{n \ln \left(\frac{d}{n}\right)-\sum_{i=1}^{n} \mathrm{E}\left[Z_{i}\right]}{\sqrt{\operatorname{Var}\left(\sum_{i=1}^{n} Z_{i}\right)}},
$$

so that $\Lambda \geq d_{\Lambda}$ implies that $\mathbb{S} \geq d$.

In the remainder of this paper, the choice of $\Lambda$ will be dependent on the time horizon $n$. To indicate this dependence, we introduce the notation $\Lambda_{n}$ for the used conditioning variable $\Lambda$. 


\subsection{Generalization to sums of lognormals with a stochastic time horizon}

Suppose that $\mathbb{S}$ is a sum of lognormal variables with a stochastic time horizon $T$

$$
\mathbb{S}=\sum_{i=1}^{T} \alpha_{i} e^{Z_{i}}
$$

with $\alpha_{i} \in \mathbb{R}, T$ a stochastic variable with life time probability distribution $F_{T}(t)$ and $Z_{i} \sim$ $N\left(\mathrm{E}\left[Z_{i}\right], \sigma_{Z_{i}}\right)$ independent of $T$. Using the tower property for conditional expectations, we can calculate the stop-loss premium of $\mathbb{S}$ as follows

$$
\begin{aligned}
\pi(\mathbb{S}, d)=\mathrm{E}\left[(\mathbb{S}-d)_{+}\right] & =\mathrm{E}\left[\left(\sum_{i=1}^{T} \alpha_{i} e^{Z_{i}}-d\right)_{+}\right] \\
& =\mathrm{E}_{T}\left[\mathrm{E}\left[\left(\sum_{i=1}^{T} \alpha_{i} e^{Z_{i}}-d\right)_{+} \mid T\right]\right] \\
& =\sum_{j=1}^{\infty} \operatorname{Pr}(T=j) \mathrm{E}\left[\left(\sum_{i=1}^{j} \alpha_{i} e^{Z_{i}}-d\right)_{+}\right] \\
& =\sum_{j=1}^{\infty} \operatorname{Pr}(T=j) \pi\left(\mathbb{S}_{j}, d\right),
\end{aligned}
$$

with

$$
\mathbb{S}_{j}=\sum_{i=1}^{j} \alpha_{i} e^{Z_{i}}
$$

Remark that in practical applications the infinite time horizon is often replaced by a finite number. It is straightforward to obtain a lower bound, denoted as $\pi^{\ell b}(\mathbb{S}, d, \boldsymbol{\Lambda})$, by looking at the combination

$$
\pi^{\ell b}(\mathbb{S}, d, \boldsymbol{\Lambda})=\sum_{j=1}^{\infty} \operatorname{Pr}(T=j) \pi^{\ell b}\left(\mathbb{S}_{j}, d, \Lambda_{j}\right)
$$

with $\boldsymbol{\Lambda}=\Lambda_{1}, \Lambda_{2}, \ldots$ and $\pi^{\ell b}\left(\mathbb{S}_{j}, d, \Lambda_{j}\right)$ given by (27) for $n=j$. The same reasoning can be followed for obtaining the comonotonic upper bound $\pi^{c u b}(\mathbb{S}, d)$, the improved comonotonic upper bound $\pi^{i c u b}(\mathbb{S}, d, \boldsymbol{\Lambda})$ and the partially exact/comonotonic upper bound $\pi^{p e c u b}(\mathbb{S}, d, \boldsymbol{\Lambda})$.

For each term $\pi\left(\mathbb{S}_{j}, d\right)$ in the sum $(33)$ we can take the minimum of two or more of the above defined upper bounds. We propose two upper bounds based on this simple idea.

The first bound takes each time the minimum of the error term (7) independent of the retention and the error term (15) dependent on the retention. Combining this with the stop-loss premium of the lower bound $\mathbb{S}^{\ell}$ results in the following upper bound

$$
\pi^{e m u b}(\mathbb{S}, d, \boldsymbol{\Lambda})=\pi^{\ell b}(\mathbb{S}, d, \boldsymbol{\Lambda})+\sum_{j=1}^{\infty} \operatorname{Pr}(T=j) \min \left(\frac{1}{2} \mathrm{E}\left[\sqrt{\operatorname{Var}\left[\mathbb{S}_{j} \mid \Lambda_{j}\right]}\right], \varepsilon\left(d_{\Lambda_{j}}\right)\right) .
$$


Calculating for each term the minimum of all the presented upper bounds

$\pi^{m i n}(\mathbb{S}, d, \boldsymbol{\Lambda})=\sum_{j=1}^{\infty} \operatorname{Pr}(T=j) \min \left(\pi^{c u b}\left(\mathbb{S}_{j}, d\right), \pi^{i c u b}\left(\mathbb{S}_{j}, d, \Lambda_{j}\right), \pi^{\text {pecub }}\left(\mathbb{S}_{j}, d, \Lambda_{j}\right), \pi^{e m u b}\left(\mathbb{S}_{j}, d, \Lambda_{j}\right)\right)$

will of course provide the best possible upper bound.

Remarkt that $\pi^{e m u b}\left(\mathbb{S}_{j}, d, \Lambda_{j}\right)=\pi^{\ell b}\left(\mathbb{S}_{j}, d, \Lambda_{j}\right)+\min \left(\frac{1}{2} \mathrm{E}\left[\sqrt{\operatorname{Var}\left[\mathbb{S}_{j} \mid \Lambda_{j}\right]}\right], \varepsilon\left(d_{\Lambda_{j}}\right)\right)$.

\section{Application to life contingencies}

In this section, we will adapt the different lower and upper bounds presented above, to the case of life contingencies, and we will compare the performance in a numerical illustration. We consider the random variable $\mathbb{S}_{n}$ which is defined as the present value of a series of $n$ deterministic non-negative payment obligations $\alpha_{1}, \alpha_{2}, \ldots, \alpha_{n}$ due at times $1,2, \ldots, n$, respectively:

$$
\mathbb{S}_{n}=\sum_{i=1}^{n} \alpha_{i} e^{-Y(i)} \stackrel{n o t}{=} \sum_{i=1}^{n} \alpha_{i} e^{Z_{i}}
$$

where the stochastic variables $Y(i)$ are defined as $Y(i):=Y_{1}+Y_{2}+\cdots+Y_{i}$. The random variables $Y_{i}$ represent the stochastic continuous compounded rate of return over the period $[i-1, i]$ and $e^{-Y(i)}$ is the random discount factor over the period $[0, i]$.

We will assume that the yearly returns $Y_{i}$ are i.i.d. normally distributed with mean $\mu=0.07$ and volatility $\sigma=0.1$. Notice that $\mathbb{S}_{n}$ is a random variable of the general type defined in (18).

In order to compute the lower and upper bounds for the stop-loss premia, we consider as conditioning random variable $\Lambda_{n}=\sum_{i=1}^{n} \gamma_{i} Z_{i}$, with in the 'Taylor-based' case $\gamma_{i}$ given by

$$
\gamma_{i}=\alpha_{i} e^{\mathrm{E}\left[Z_{i}\right]}, \quad i=1, \ldots, n
$$

and in the 'maximal variance' case $\gamma_{i}$ given by

$$
\gamma_{i}=\alpha_{i} e^{\mathrm{E}\left[Z_{i}\right]+\frac{1}{2} \sigma_{Z_{i}}^{2}}, \quad i=1, \ldots, n .
$$

The corresponding decomposition variables are respectively equal to $d-\sum_{i=1}^{n} \alpha_{i} e^{\mathrm{E}\left[Z_{i}\right]}\left(1-\mathrm{E}\left[Z_{i}\right]\right)$ and $d-\sum_{i=1}^{n} \alpha_{i} e^{\mathrm{E}\left[Z_{i}\right]+\frac{1}{2} \sigma_{Z_{i}}^{2}}\left(1-\mathrm{E}\left[Z_{i}\right]-\frac{1}{2} \sigma_{Z_{i}}^{2}\right)$. For the numerical illustrations in this section we present each time the one which provides the best result.

Notice that $\mathrm{E}\left[Z_{i}\right], \sigma_{Z_{i}}^{2}$ and $r_{i}$ are given by

$$
\begin{aligned}
\mathrm{E}\left[Z_{i}\right] & =-i \mu \\
\sigma_{Z_{i}}^{2} & =i \sigma^{2}
\end{aligned}
$$

and

$$
r_{i}=\frac{\sum_{j=1}^{i} \sum_{k=j}^{n} \gamma_{k}}{\sqrt{i \sum_{j=1}^{n}\left(\sum_{k=j}^{n} \gamma_{k}\right)^{2}}}
$$


Remark that the correlation coefficients $r_{i}$ are positive, so that the formulae (26) and (27) can be applied.

The 'maximal variance' conditioning variable with $\gamma_{i}=\alpha_{i} e^{\mathrm{E}\left[Z_{i}\right]+\frac{1}{2} \sigma_{Z_{i}}^{2}}$ performs better far in the tail. So for high values of $d$ the different bounds based on this conditioning variable approximate more accurate the real value of the stop-loss premium than the approximations using the 'Taylorbased' conditioning variable. The 'geometric average' conditioning variable performs in general sligthly worse in comparison with the two other random variables for this kind of applications.

Now we will compare the performance of the different bounds that were presented in Sections 2, 3 and 4: the lower bound $\pi^{\ell b}\left(\mathbb{S}_{n}, d, \Lambda\right)(\mathrm{LB})$, the comonotonic upper bound $\pi^{c u b}\left(\mathbb{S}_{n}, d\right)$ (CUB), the improved comonotonic upper bound $\pi^{i c u b}\left(\mathbb{S}_{n}, d, \Lambda\right)$ (ICUB), the upper bound based on the lower bound $\pi^{e u b}\left(\mathbb{S}_{n}, d, \Lambda\right)(\mathrm{EUB})$ and $\pi^{d e u b}\left(\mathbb{S}_{n}, d, \Lambda\right)$ (DEUB) and the partially exact/comonotonic upper bound $\pi^{\text {pecub }}\left(\mathbb{S}_{n}, d, \Lambda\right)$ (PECUB). For applications with a stochastic time horizon $N$ we also consider the two combination bounds $\pi^{e m u b}\left(\mathbb{S}_{N}, d, \boldsymbol{\Lambda}\right)(\mathrm{EMUB})$ and $\pi^{\min }\left(\mathbb{S}_{N}, d, \boldsymbol{\Lambda}\right)(\mathrm{MIN})$.

We will compare the different lower and upper bounds for the stop-loss premiums with the values obtained by Monte-Carlo simulation (MC). The simulation results are based on generating $50 \times 1000000$ paths. For each estimate we computed the standard error (s.e.). As is well-known, the (asymptotic) 95\% confidence interval is given by the estimate plus or minus 1.96 times the standard error. The estimates obtained from this time-consuming simulation will serve as benchmark. The random paths are based on antithetic variables in order to reduce the variance of the Monte-Carlo estimates.

We will apply the above derived bounds for two kind of life insurance applications. A life annuity may be defined as a series of periodic payments where each payment will actually be made only if a designated life is alive at the time the payment is due. Let us consider a person aged $x$ years, also called a life aged $x$ and denoted by $(x)$. We denote his or her future lifetime by $T_{x}$. Thus $x+T_{x}$ will be the age of death of the person. The future lifetime $T_{x}$ is a random variable with a probability distribution function

$$
G_{x}(t)=\operatorname{Pr}\left[T_{x} \leq t\right]={ }_{t} q_{x}, \quad t \geq 0 .
$$

The function $G_{x}$ represents the probability that the person will die within $t$ years, for any fixed $t$. We assume that $G_{x}$ is known. We define $K_{x}=\left\lfloor T_{x}\right\rfloor$, the number of completed future years lived by $(x)$, or the curtate future lifetime of $(x)$, where $\lfloor$.$\rfloor is the floor function, i.e. \lfloor x\rfloor$ is the largest integer less than or equal to $x$. The probability distribution of the integer valued random variable $K_{x}$ is given by

$$
\operatorname{Pr}\left(K_{x}=k\right)=\operatorname{Pr}\left(k \leq T_{x}<k+1\right)={ }_{k+1} q_{x}-{ }_{k} q_{x}={ }_{k \mid} q_{x}, \quad k=0,1, \ldots
$$

Further, the ultimate age of the life table is denoted by $\omega$, this means that $\omega-x$ is the first remaining lifetime of $(x)$ for which $\omega_{\omega-x} q_{x}=1$, or equivalently, $G_{x}^{-1}(1)=\omega-x$. 
We assume in this section that the distribution of the remaining lifetime belongs to the GompertzMakeham family. Analytic life tables are defined for all ages $x \geq 0$. In Makeham's model ${ }^{1} l_{x}$, the number of persons alive at age $x$, is given by

$$
l_{x}=a s^{x} g^{c^{x}},
$$

where $a>0,0<s<1,0<g<1$ and $c>1$. Makeham's life table results from the force of mortality function

$$
\mu_{\xi}=\alpha+\beta c^{\xi}
$$

where $\alpha$ is a constant component, interpreted as capturing accident hazard, and $\beta c^{\xi}$ is a variable component capturing the hazard of aging. The relationship between (35) and (36) is given by

$$
a:=l_{0} e^{\frac{\beta}{\log c}}, s:=e^{-\alpha} \text { and } g:=e^{-\frac{\beta}{\log c}} .
$$

See Bowers et al. (1996) for more details.

For generating one random variate from Makeham's law, we use the composition method (Devroye, 1986) and perform the following steps

(a) Generate $G$ from the Gompertz's law by the well-known inversion method

(b) Generate $E$ for the exponential(1) distribution

(c) Retain $T=\min (E / \alpha, G)$,

where $\alpha=-\log s$, see (37).

In the remainder of this paper, we will always use the standard actuarial notation:

$$
\operatorname{Pr}\left[T_{x}>t\right]={ }_{t} p_{x}, \operatorname{Pr}\left[T_{x}>1\right]=p_{x}, \operatorname{Pr}\left[T_{x} \leq t\right]={ }_{t} q_{x}, \quad \operatorname{Pr}\left[T_{x} \leq 1\right]=q_{x}
$$

First we consider a whole life annuity on a life $(x)$ which pays an amount of 1 at the end of each year, provided the insured is still alive at that time. Assume that the discounting is performed with a random interest rate. The present value at policy issue of the future payments is denoted ${ }^{2}$ by $\mathbb{S}_{x}^{\text {policy }}$ and equals the sum of the present values of the payments in the respective years:

$$
\mathbb{S}_{x}^{\text {policy }}=\sum_{i=1}^{\lfloor\omega-x\rfloor} 1_{\left\{T_{x}>i\right\}} e^{-Y(i)}=\sum_{i=1}^{K_{x}} e^{-Y(i)},
$$

where $1_{\{.\}}$denotes the indicator function.

\footnotetext{
${ }^{1}$ we use the Belgian analytic life tables MR and FR for life annuity valuation, with corresponding constants for $l_{0}$, the number of newborns, equal to 1000000 . For males: $a=1000266.63, s=0.999441703848, g=$ $0.999733441115, c=1.101077536030$, and for females: $a=1000048.56, s=0.999669730966, g=0.999951440171$, $c=1.116792453830$.

${ }^{2}$ in literature denoted by $a_{K}, K \geq 0$.
} 
The stop-loss premium for $\mathbb{S}_{x}^{\text {policy }}$ can be calculated as

$$
\mathrm{E}\left[\left(\mathbb{S}_{x}^{\text {policy }}-d\right)_{+}\right]=\sum_{i=1}^{\lfloor\omega-x\rfloor} i \mid q_{x} \mathrm{E}\left[\left(\tilde{\mathbb{S}}_{i}-d\right)_{+}\right],
$$

where $\widetilde{\mathbb{S}}_{i}$ is a special case of $\mathbb{S}_{i}$ defined in $(34)$ for unit payments $\left(\left(\alpha_{1}, \ldots, \alpha_{i}\right)=(1, \ldots, 1)\right)$. We will approximate $\mathrm{E}\left[\left(\tilde{\mathbb{S}}_{i}-d\right)_{+}\right]$by one of the derived bounds.

Consider a 65-years old male person. The different lower and upper bounds for the stop-loss premium of a whole life annuity due of 1 payable at the end of each year (annuity-immediate) while (65) survives are compared in Table 1. For the retentions $d=5,10$ and 15 the upper bound $\pi^{\text {min }}\left(\mathbb{S}_{x}^{\text {policy }}, d, \boldsymbol{\Lambda}\right)$ really improves the comonotonic and improved comonotonic upper bound. For the extreme cases the values are more or less the same. The lower bound is very close to the real stop-loss premium.

\begin{tabular}{|l|l|l|l|l|l|l|l|}
\hline & $d=0$ & $d=5$ & $d=10$ & $d=15$ & $d=20$ & $d=25$ & $d=30$ \\
\hline LB & 9.3196 & 4.6191 & 1.2269 & 0.1737 & 0.0207 & 0.0026 & 0.0004 \\
MC & 9.3196 & 4.6191 & 1.2304 & 0.1739 & 0.0216 & 0.0026 & 0.0004 \\
$\left(\right.$ s.e. $\left.\times 10^{5}\right)$ & & $(8.49)$ & $(5.48)$ & $(0.51)$ & $(0.19)$ & $(0.01)$ & $(0.002)$ \\
ICUB & 9.3196 & 4.6238 & 1.3277 & 0.2530 & 0.0454 & 0.0088 & 0.0019 \\
CUB & 9.3196 & 4.6244 & 1.3389 & 0.2610 & 0.0480 & 0.0095 & 0.0021 \\
EMUB & 9.3196 & 4.6197 & 1.2400 & 0.2145 & 0.0718 & 0.0545 & 0.0522 \\
PECUB & 9.3196 & 4.6219 & 1.2839 & 0.2381 & 0.0451 & 0.0088 & 0.0019 \\
MIN & 9.3196 & 4.6195 & 1.2385 & 0.2070 & 0.0444 & 0.0088 & 0.0019 \\
\hline
\end{tabular}

Table 1: Approximations for stop-loss premia with retention $d$ of $\mathbb{S}_{x}^{\text {policy }}$.

In a second application we consider a portfolio of $N_{0}$ homogeneous life annuity contracts for which future lifetimes of the insureds $T_{x}^{(1)}, T_{x}^{(2)}, \ldots, T_{x}^{\left(N_{0}\right)}$ are assumed to be independent. Then the insurer faces two risks: mortality risk and investment risk. Note that from the Law of Large Numbers the mortality risk decreases with the number of policies $N_{0}$ while the investment risk remains the same (each of the policies is exposed to the same investment risk). Thus for sufficiently large $N_{0}$ the stop-loss premium of the portfolio can be expressed as follows

$$
\begin{aligned}
\mathrm{E}\left[\left(\sum_{i=1}^{\lfloor\omega-x\rfloor} N_{i} e^{-Y(i)}-d\right)_{+}\right] & =\mathrm{E}\left[N_{0}\left(\sum_{i=1}^{\lfloor\omega-x\rfloor} \frac{N_{i}}{N_{0}} e^{-Y(i)}-\frac{d}{N_{0}}\right)_{+}\right] \\
& \approx N_{0} \mathrm{E}\left[\left(\sum_{i=1}^{\lfloor\omega-x\rfloor} i p_{x} e^{-Y(i)}-\frac{d}{N_{0}}\right)_{+}\right]
\end{aligned}
$$

where $N_{i}$ denotes a number of survivals after $i$-th year. Hence in the case of large portfolios of life annuities it suffices to compute stop-loss premiums of an "average" portfolio $\mathbb{S}_{x}^{\text {average }}$ given by

$$
\mathbb{S}_{x}^{\text {average }}=\sum_{i=1}^{\lfloor\omega-x\rfloor} i p_{x} e^{-Y(i)}
$$


what has exactly the form of $(34)$ with $\alpha_{i}={ }_{i} p_{x}(i=1, \ldots,\lfloor\omega-x\rfloor)$.

Table 2 shows the results for the stop-loss premium $\mathrm{E}\left[\left(\mathbb{S}_{x}^{\text {average }}-d\right)_{+}\right]$with different retentions $d$. Again the lower bound approach approximates the exact stop-loss premiums extremely well. The results for the upper bounds are in line with the previous ones. Note that for very high values of $d$ the differences become larger, however these cases don't represent any practical importance.

\begin{tabular}{|l|l|l|l|l|}
\hline & $d=0$ & $d=5$ & $d=10$ & $d=15$ \\
\hline LB & 9.3196 & 4.3200 & 0.5533 & 0.0193 \\
MC & 9.3196 & 4.3200 & 0.5543 & 0.0197 \\
$\left(\right.$ s.e. $\left.\times 10^{5}\right)$ & & $(0.37)$ & $(0.13)$ & $(0.035)$ \\
ICUB & 9.3196 & 4.3227 & 0.7076 & 0.0523 \\
CUB & 9.3196 & 4.3233 & 0.7217 & 0.0559 \\
EUB & 9.3751 & 4.3755 & 0.6090 & 0.0749 \\
DEUB & 9.3196 & 4.3202 & 0.5784 & 0.0744 \\
PECUB & 9.3196 & 4.3219 & 0.6515 & 0.0522 \\
\hline
\end{tabular}

Table 2: Approximations for stop-loss premia with retention $d$ of $\mathbb{S}_{x}^{\text {average }}$.

Remark that only for EUB the error term is independent of the retention and therefore in both tables all values for $d=0$, except these for EUB, are identical and equal to 9.3196. This follows from the fact that in this case the expected value of $\mathbb{S}_{x}^{p o l i c y}$ equals the expected value of $\mathbb{S}_{x}^{\text {average }}$. Note also that the values in Table 1 are typically larger than the corresponding values in Table 2. This is not surprising. From Hoedemakers et al. (2004) (Example 1) it immediately follows that $\mathbb{S}_{x}^{\text {average }} \leq_{c x} \mathbb{S}_{x}^{\text {policy }}$ and hence for any retention $d>0$ one has

$$
\mathrm{E}\left[\left(\mathbb{S}_{x}^{\text {average }}-d\right)_{+}\right] \leq \mathrm{E}\left[\left(\mathbb{S}_{x}^{\text {policy }}-d\right)_{+}\right]
$$

\section{$7 \quad$ Summary and conclusions}

In this paper we generalized some methodologies for estimating the stop-loss premiums of strongly dependent random variables. We started with the comonotonic approximations of Dhaene et al. (2002a) and the upper bound obtained by adding an error term to the lower bound of Rogers and Shi (1995). We explained how these bounds can be improved by decomposing an integral formula for the stop-loss premium into two parts: one can be easily solved analytically, the other part can be approximated by one of the comonotonic upper bounds.

We apply all the methods to an average portfolio of life annuities (when mortality risk is assumed to be fully diversified) and to a single life annuity. In the latter case it is possible to decompose the value of the stop-loss premium by conditioning and apply the best (smallest) upper bound on each of the component separately. We provide a number of numerical illustrations which show that the decomposition significantly improves the bounds. 


\section{Acknowledgements}

Tom Hoedemakers, Grzegorz Darkiewicz and Jan Dhaene acknowledge the financial support of the Onderzoeksfonds K.U.Leuven (GOA/02: Actuariële, financiële en statistische aspecten van afhankelijkheden in verzekerings- en financiële portefeuilles).

Michèle Vanmaele would like to acknowledge the financial support by the BOF-project 001104599 of the Ghent University.

\section{Corresponding author}

Tom Hoedemakers

Catholic University of Leuven

Department of Applied Economics

Naamsestraat 69, B-3000 Leuven, Belgium

tel: ++32 16 32.67.61, fax: ++32 1632.67 .32

email: tom.hoedemakers@econ.kuleuven.ac.be 


\section{Appendix}

\section{A - Upper bound based on lower bound}

In the following we shall derive an easily computable expression for (15).

The second expectation term in the product (15) equals, when denoting by $F_{\Lambda}(\cdot)$ the normal cumulative distribution function of $\Lambda$,

$$
\mathrm{E}\left[1_{\left\{\Lambda<d_{\Lambda}\right\}}\right]=0 \cdot P\left(\Lambda \geq d_{\Lambda}\right)+1 \cdot P\left(\Lambda<d_{\Lambda}\right)=F_{\Lambda}\left(d_{\Lambda}\right)=\Phi\left(d_{\Lambda}^{*}\right) .
$$

The first expectation term in the product (15) can be expressed as

$$
\mathrm{E}\left[\operatorname{Var}(\mathbb{S} \mid \Lambda) 1_{\left\{\Lambda<d_{\Lambda}\right\}}\right]=\mathrm{E}\left[\mathrm{E}\left[\mathbb{S}^{2} \mid \Lambda\right] 1_{\left\{\Lambda<d_{\Lambda}\right\}}\right]-\mathrm{E}\left[(\mathrm{E}[\mathbb{S} \mid \Lambda])^{2} 1_{\left\{\Lambda<d_{\Lambda}\right\}}\right] .
$$

Now consider the second term of the right-hand side of (39)

$$
\mathrm{E}\left[(\mathrm{E}[\mathbb{S} \mid \Lambda])^{2} 1_{\left\{\Lambda<d_{\Lambda}\right\}}\right]=\int_{-\infty}^{d_{\Lambda}}(\mathrm{E}[\mathbb{S} \mid \Lambda=\lambda])^{2} d F_{\Lambda}(\lambda)
$$

According to (21) and using the notation $Z_{i j}$ introduced in Section 5.4 we can express (40) as

$$
\begin{aligned}
\mathrm{E} & {\left[(\mathrm{E}[\mathrm{S} \mid \Lambda])^{2} 1_{\left\{\Lambda<d_{\Lambda}\right\}}\right] } \\
& =\int_{-\infty}^{d_{\Lambda}}\left(\sum_{i=1}^{n} \mathrm{E}\left[X_{i} \mid \Lambda=\lambda\right]\right)^{2} d F_{\Lambda}(\lambda) \\
& =\int_{-\infty}^{d_{\Lambda}}\left(\sum_{i=1}^{n} \alpha_{i} e^{\mathrm{E}\left[Z_{i}\right]+r_{i} \sigma_{Z_{i}} \Phi^{-1}(v)+\frac{1}{2}\left(1-r_{i}^{2}\right) \sigma_{Z_{i}}^{2}}\right)^{2} d F_{\Lambda}(\lambda) \\
& =\int_{-\infty}^{d_{\Lambda}} \sum_{i=1}^{n} \sum_{j=1}^{n} \alpha_{i} \alpha_{j} e^{\mathrm{E}\left[Z_{i j}\right]+\left(r_{i} \sigma_{Z_{i}}+r_{j} \sigma_{Z_{j}}\right) \Phi^{-1}(v)+\frac{1}{2}\left\{\left(1-r_{i}^{2}\right) \sigma_{Z_{i}}^{2}+\left(1-r_{j}^{2}\right) \sigma_{Z_{j}}^{2}\right\}} d F_{\Lambda}(\lambda) \\
& =\sum_{i=1}^{n} \sum_{j=1}^{n} \alpha_{i} \alpha_{j} e^{\mathrm{E}\left[Z_{i j}\right]+\frac{1}{2}\left\{\left(1-r_{i}^{2}\right) \sigma_{Z_{i}}^{2}+\left(1-r_{j}^{2}\right) \sigma_{Z_{j}}^{2}\right\}} \int_{-\infty}^{d_{\Lambda}} e^{\left(r_{i} \sigma_{Z_{i}}+r_{j} \sigma_{Z_{j}}\right) \Phi^{-1}(v)} d F_{\Lambda}(\lambda) .
\end{aligned}
$$

Next, applying Lemma 2 to (41) with $a=r_{i} \sigma_{Z_{i}}+r_{j} \sigma_{Z_{j}}$ yields

$$
\begin{aligned}
\mathrm{E} & {\left[(\mathrm{E}[\mathbb{S} \mid \Lambda])^{2} 1_{\left\{\Lambda<d_{\Lambda}\right\}}\right] } \\
& =\sum_{i=1}^{n} \sum_{j=1}^{n} \alpha_{i} \alpha_{j} e^{\mathrm{E}\left[Z_{i j}\right]+\frac{1}{2}\left(\sigma_{Z_{i}}^{2}+\sigma_{Z_{j}}^{2}+2 r_{i} r_{j} \sigma_{Z_{i}} \sigma_{Z_{j}}\right)} \Phi\left(d_{\Lambda}^{*}-\left(r_{i} \sigma_{Z_{i}}+r_{j} \sigma_{Z_{j}}\right)\right) .
\end{aligned}
$$

Now consider the first term of the right-hand side of $(39), \mathrm{E}\left[\mathrm{E}\left[\mathbb{S}^{2} \mid \Lambda\right] 1_{\left\{\Lambda<d_{\Lambda}\right\}}\right]$. The term $\mathrm{E}\left[\mathbb{S}^{2} \mid \Lambda\right]$ 
is given by (29). By applying (30) with $a=r_{i j} \sigma_{Z_{i j}}=r_{i} \sigma_{Z_{i}}+r_{j} \sigma_{Z_{j}}$, and simplifying, we obtain

$$
\begin{aligned}
\mathrm{E} & {\left[\mathrm{E}\left[\mathbb{S}^{2} \mid \Lambda\right] 1_{\left\{\Lambda<d_{\Lambda}\right\}}\right] } \\
& =\sum_{i=1}^{n} \sum_{j=1}^{n} \int_{-\infty}^{d_{\Lambda}} \alpha_{i} \alpha_{j} e^{\mathrm{E}\left[Z_{i j}\right]+r_{i j} \sigma_{Z_{i j}} \Phi^{-1}(v)+\frac{1}{2}\left(1-r_{i j}^{2}\right) \sigma_{Z_{i j}}^{2}} d F_{\Lambda}(\lambda) \\
& =\sum_{i=1}^{n} \sum_{j=1}^{n} \alpha_{i} \alpha_{j} e^{\mathrm{E}\left[Z_{i j}\right]+\frac{1}{2}\left(1-r_{i j}^{2}\right) \sigma_{Z_{i j}}^{2}} \int_{-\infty}^{d_{\Lambda}} e^{r_{i j} \sigma_{Z_{i j}} \Phi^{-1}(v)} d F_{\Lambda}(\lambda) \\
& =\sum_{i=1}^{n} \sum_{j=1}^{n} \alpha_{i} \alpha_{j} e^{\mathrm{E}\left[Z_{i j}\right]+\frac{1}{2}\left(1-r_{i j}^{2}\right) \sigma_{Z_{i j}}^{2}+\frac{r_{i j}^{2} \sigma_{Z_{i j}}^{2}}{2} \Phi\left(d_{\Lambda}^{*}-r_{i j} \sigma_{Z_{i j}}\right)} \\
& =\sum_{i=1}^{n} \sum_{j=1}^{n} \alpha_{i} \alpha_{j} e^{\mathrm{E}\left[Z_{i j}\right]+\frac{\sigma_{Z_{i j}}^{2}}{2}} \Phi\left(d_{\Lambda}^{*}-\left(r_{i} \sigma_{Y_{i}}+r_{j} \sigma_{Y_{j}}\right)\right) .
\end{aligned}
$$

Combining (43) and (42) into (39), and then substituting (38) and (39) into (15) we get the following expression for the error bound $\varepsilon\left(d_{\Lambda}\right)(15)$ :

$$
\begin{aligned}
\varepsilon\left(d_{\Lambda}\right)= & \frac{1}{2}\left(\Phi\left(d_{\Lambda}^{*}\right)\right)^{\frac{1}{2}}\left\{\sum _ { i = 1 } ^ { n } \sum _ { j = 1 } ^ { n } \alpha _ { i } \alpha _ { j } \left[e^{\mathrm{E}\left[Z_{i j}\right]+\frac{\sigma_{Z_{i j}}^{2}}{2}} \Phi\left(d_{\Lambda}^{*}-\left(r_{i} \sigma_{Z_{i}}+r_{j} \sigma_{Z_{j}}\right)\right)\right.\right. \\
& \left.\left.-e^{\mathrm{E}\left[Z_{i j}\right]+\frac{1}{2}\left(\sigma_{Z_{i}}^{2}+\sigma_{Z_{j}}^{2}+2 r_{i} r_{j} \sigma_{Z_{i}} \sigma_{Z_{j}}\right)} \Phi\left(d_{\Lambda}^{*}-\left(r_{i} \sigma_{Z_{i}}+r_{j} \sigma_{Z_{j}}\right)\right)\right]\right\}^{\frac{1}{2}} \\
= & \frac{1}{2}\left(\Phi\left(d_{\Lambda}^{*}\right)\right)^{\frac{1}{2}} \times \\
& \times\left\{\sum_{i=1}^{n} \sum_{j=1}^{n} \alpha_{i} \alpha_{j} e^{\mathrm{E}\left[Z_{i j}\right]} \Phi\left(d_{\Lambda}^{*}-\left(r_{i} \sigma_{Z_{i}}+r_{j} \sigma_{Z_{j}}\right)\right)\left(e^{\frac{1}{2}\left(\sigma_{Z_{i}}^{2}+\sigma_{Z_{j}}^{2}+2 \sigma_{Z_{i} Z_{j}}\right)}-e^{\frac{1}{2}\left(\sigma_{Z_{i}}^{2}+\sigma_{Z_{j}}^{2}+2 r_{i} r_{j} \sigma_{Z_{i}} \sigma_{Z_{j}}\right)}\right)\right\}^{\frac{1}{2}} \\
= & \frac{1}{2}\left(\Phi\left(d_{\Lambda}^{*}\right)\right)^{\frac{1}{2}} \times \\
& \times\left\{\sum _ { i = 1 } ^ { n } \sum _ { j = 1 } ^ { n } \alpha _ { i } \alpha _ { j } e ^ { \mathrm { E } [ Z _ { i j } ] + \frac { 1 } { 2 } ( \sigma _ { Z _ { i } } ^ { 2 } + \sigma _ { Z _ { j } } ^ { 2 } ) } \Phi ( d _ { \Lambda } ^ { * } - ( r _ { i } \sigma _ { Z _ { i } } + r _ { j } \sigma _ { Z _ { j } } ) ) \left(e^{\left.\left.\sigma_{Z_{i} Z_{j}}-e^{\sigma_{Z_{i}} \sigma_{Z_{j}} r_{i} r_{j}}\right)\right\}^{\frac{1}{2}}} .\right.\right.
\end{aligned}
$$




\section{B - Partially exact/comonotonic upper bound}

Applying Lemma 2 with $a=r_{i} \sigma_{Z_{i}}$, and using (21), we can express the second term $I_{2}$ in (11) in closed-form:

$$
\begin{aligned}
& \int_{d_{\Lambda}}^{+\infty} \mathrm{E}[\mathbb{S}-d \mid \Lambda=\lambda] d F_{\Lambda}(\lambda) \\
& =\int_{d_{\Lambda}}^{+\infty} \mathrm{E}[\mathbb{S} \mid \Lambda=\lambda] d F_{\Lambda}(\lambda)-d\left(1-F_{\Lambda}\left(d_{\Lambda}\right)\right) \\
& =\sum_{i=1}^{n} \alpha_{i} e^{\mathrm{E}\left[Z_{i}\right]+\frac{1}{2}\left(1-r_{i}^{2}\right) \sigma_{Z_{i}}^{2}} \int_{d_{\Lambda}}^{+\infty} e^{r_{i} \sigma_{Z_{i}} \Phi^{-1}(v)} d F_{\Lambda}(\lambda)-d\left(1-\Phi\left(d_{\Lambda}^{*}\right)\right) \\
& =\sum_{i=1}^{n} \alpha_{i} e^{\mathrm{E}\left[Z_{i}\right]+\frac{\sigma_{Z_{i}}^{2}}{2}} \Phi\left(r_{i} \sigma_{Z_{i}}-d_{\Lambda}^{*}\right)-d \Phi\left(-d_{\Lambda}^{*}\right) .
\end{aligned}
$$

Substituting (22) in (17) we end up with the following upper bound of $I_{1}$ similar to (25) but now with an integral from zero to $\Phi\left(d_{\Lambda}^{*}\right)$ :

$$
\begin{aligned}
& \int_{-\infty}^{d_{\Lambda}} \mathrm{E}\left[(\mathbb{S}-d)_{+} \mid \Lambda=\lambda\right] d F_{\Lambda}(\lambda) \\
& \leq \int_{-\infty}^{d_{\Lambda}} \mathrm{E}\left[\left(\mathbb{S}^{u}-d\right)_{+} \mid \Lambda=\lambda\right] d F_{\Lambda}(\lambda) \\
& =\int_{0}^{\Phi\left(d_{\Lambda}^{*}\right)} \mathrm{E}\left[\left(\mathbb{S}^{u}-d\right)_{+} \mid V=v\right] d v \\
& =\sum_{i=1}^{n} \alpha_{i} e^{\mathrm{E}\left[Z_{i}\right]+\frac{1}{2} \sigma_{Z_{i}}^{2}\left(1-r_{i}^{2}\right)} \int_{0}^{\Phi\left(d_{\Lambda}^{*}\right)} e^{r_{i} \sigma_{Z_{i}} \Phi^{-1}(v)} \Phi\left(\operatorname{sign}\left(\alpha_{i}\right) \sqrt{1-r_{i}^{2}} \sigma_{Z_{i}}-\Phi^{-1}\left(F_{\mathbb{S}^{u} \mid V=v}(d)\right)\right) d v \\
& -d\left(\Phi\left(d_{\Lambda}^{*}\right)-\int_{0}^{\Phi\left(d_{\Lambda}^{*}\right)} F_{\mathbb{S}^{u} \mid V=v}(d) d v\right) .
\end{aligned}
$$

where we recall that $d_{\Lambda}^{*}$ is defined as in (30), and the cumulative distribution $F_{\mathbb{S}^{u}}(d)$ is, according to $(24)$, determined by

$$
\sum_{i=1}^{n} \alpha_{i} e^{\mathrm{E}\left[Z_{i}\right]+r_{i} \sigma_{Z_{i}} \Phi^{-1}(v)+\operatorname{sign}\left(\alpha_{i}\right) \sqrt{1-r_{i}^{2}} \sigma_{Z_{i}} \Phi^{-1}\left(F_{\mathbb{S} u}(d \mid V=v)\right)}=d .
$$

Finally, adding (45) to the exact part (44) of the decomposition (11) results in the partially exact/comonotonic upper bound. 


\section{References}

[1] Bowers, N.L., Gerber, H.U., Hickman, J.C., Jones, D.A. and Nesbitt, C.J. (1986). Actuarial mathematics. Schaumburg, Ill.: Society of Actuaries.

[2] Curran, M. (1994). Valuing Asian and portfolio options by conditioning on the geometric mean price. Management Science, 40(12), 1705-1711.

[3] De Vylder, F. and Goovaerts, M.J. (1982). Upper and lower bounds on stop-loss premiums in case of known expectation and variance of the risk variable. Mitt. Verein. Schweiz. Versicherungmath., 149-164.

[4] Deelstra, G., Liinev, J. and Vanmaele, M. (2004). Pricing of arithmetic basket options by conditioning, Insurance: Mathematics and Economics. 34(1), 55-77.

[5] Devroye, L. (1986). Non-Uniform random variate generation, Springer-Verlag, New York.

[6] Dhaene, J., Denuit, M., Goovaerts, M.J., Kaas, R. and Vyncke, D. (2002a). The concept of comonotonicity in actuarial science and finance: theory. Insurance: Mathematics and Economics, 31(1), 3-33.

[7] Dhaene, J., Denuit, M., Goovaerts, M.J., Kaas, R. and Vyncke, D. (2002b). The concept of comonotonicity in actuarial science and finance: applications. Insurance: Mathematics and Economics, 31(2), 133-161.

[8] Hoedemakers, T., Darkiewicz, G., Dhaene, J. and Goovaerts, M.J. (2004). On the Distribution of Life Annuities with Stochastic Interest Rates. Proceedings of the Eighth International Congress on Insurance: Mathematics and Economics, Rome.

[9] Hürlimann, W. (1996). Improved analytical bounds for some risk quantities. ASTIN Bulletin, 26(2), 185-199.

[10] Hürlimann, W. (1998). On best stop-loss bounds for bivariate sums by known marginal means, variances and correlation. Mitt. Verein. Schweiz. Versicherungmath., 111-134.

[11] Jansen, K., Haezendonck, J. and Goovaerts, M.J. (1986). Upper bounds on stop-loss premiums in case of known moments up to the fourth order. Insurance: Mathematics and Economics, 5(4), 315-334.

[12] Kaas, R, Dhaene, J. and Goovaerts, M.J. (2000). Upper and lower bounds for sums of random variables. Insurance: Mathematics and Economics, 27(2), 151-168.

[13] Nielsen, J.A. and Sandmann, K. (2003). Pricing bounds on Asian options. Journal of Financial and Quantitative Analysis, 38(2).

[14] Rogers, L.C.G. and Shi, Z. (1995). The value of an Asian option. Journal of Applied Probability, 32, 1077-1088.

[15] Simon, S., Goovaerts, M.J. and Dhaene, J. (2000). An easy computable upper bound for the price of an arithmetic Asian option. Insurance: Mathematics and Economics, 26(2-3), $175-184$ 
[16] Vanduffel, S., Hoedemakers, T. and Dhaene, J., 2004. Comparing approximations for risk measures of sums of non-independent lognormal random variables. www.kuleuven.ac.be/insurance, publications.

[17] Vanmaele, M., Deelstra, G. and Liinev, J. (2004a). Approximation of stop-loss premiums involving sums of lognormals by conditioning on two random variables. Insurance: Mathematics and Economics, 35(2), 343-367.

[18] Vanmaele, M., Deelstra, G., Liinev, J., Dhaene, J. and Goovaerts M.J. (2004b). Bounds for the price of discrete arithmetic Asian options. Journal of Computational and Applied Mathematics, accepted. 